\title{
Tecnologias na Segurança Pública: prospecção sobre o uso de inteligências artificiais e outros dispositivos
}

\author{
Technologies in Public Safety: prospection about the use of artificial \\ intelligence and another devices
}

\author{
Edilson Ponciano de Lima ${ }^{1}$ \\ João Paulo Lima Santos ${ }^{1}$ \\ Vinícius José Ferro Gomes ${ }^{1}$ \\ Erivaldo Oliveira de Matos ${ }^{1}$ \\ Francisco Medson Lima Maia ${ }^{1}$ \\ Luiz André da Silva Lima ${ }^{1}$ \\ José Maurício Alencar Filho ${ }^{2}$ \\ Wagner Gonçalves Oliveira e Silva ${ }^{2}$ \\ Gesyca Patrícia da Silva Santos ${ }^{1}$ \\ ${ }^{1}$ Universidade Federal de Alagoas, Maceió, AL, Brasil \\ ${ }^{2}$ Centro Universitário Tiradentes, Maceió, AL, Brasil
}

\begin{abstract}
Resumo
Este artigo realiza uma prospecção tecnológica sobre o uso de inteligências artificiais e Internet das Coisas direcionadas à sua aplicação na Segurança Pública, apresentando tendências que poderão aprimorar as informações e desenvolver os serviços prestados pelos agentes da segurança pública no Brasil. As tendências tecnológicas são demonstradas por meio de uma prospecção tecnológica nas bases patentárias Lens, Questel-Orbit e a Derwent Index Innovation. Foram reunidos os dados sobre patentes com pesquisas bibliográficas na área, podendo ser traçado um breve panorama das tendências sobre o uso de inteligências artificiais aliadas à Internet das Coisas (IoT), resultando em dispositivos de reconhecimento facial, sensores de disparo, identificação por rádio frequência e diversos serviços que podem ser diretamente relacionados à Segurança Pública.
\end{abstract}

Palavras-chave: Segurança Pública. Internet das Coisas. RFID. Tecnologia.

\begin{abstract}
This article conducts a technological survey about the artificial intelligence and Internet of Things (IoT) directed to its application in Public Security presenting trends that can improve information and develop the services provided by public security agents in Brazil. Technological trends are demonstrated through a technological prospection inside the patent bases: Lens, Questel-Orbit and the Derwent Index Innovation. The patent data were gathered together with bibliographical researches in the area creating a brief overview of trends about the use of the artificial intelligence combined with the Internet of Things (IoT) resulting in facial recognition devices, trigger sensors, radio frequency identification and another services that can be directly related to Public Security.
\end{abstract}

Keywords: Public Safety. Internet of Things. RFID. Technology.

Área Tecnológica: Internet das Coisas. Propriedade Intelectual. Segurança. 


\section{Introdução}

Segundo o conceito apresentado por Oliveira et al. (2017), a Internet of Things (IoT) surgiu para explicar a convergência de múltiplas tecnologias que envolvem a comunicação sem fio, internet, sistemas embarcados e microeletromecânicos. Cada objeto é identificado por meio do seu sistema de software embarcado e é capaz de interoperar com a infraestrutura de internet existente. Os principais componentes de uma rede IoT podem ser, por exemplo, aparelhos celulares e eletroeletrônicos, sensores, computadores, aparelhos de televisores, ares-condicionados, lâmpadas e diversos outros dispositivos utilizados, tanto no ambiente doméstico quando na aplicação industrial. As redes de comunicação que as conectam são os sistemas computacionais que processam e fazem o uso dos dados que os dispositivos transmitem e recebem. Dessa forma, com a mobilidade e a tecnologia avançando de forma rápida e eficiente, será possível acumular dados, tanto pessoais como industriais, em grandes quantidades (Big Data) e trabalhá-los a favor de decisões mais exatas em grandes empresas até oferecer uma mais vida confortável para indivíduos em seus lares.

Dentro do conceito de Internet das Coisas, o uso de um alto volume de informações gera diretrizes para a tomada de decisão, o que foi conceituado como "Big Data". Segundo Taurion (2013), "Big Data" não trata somente volume de dados, mas da necessidade desses dados não estruturados serem validados nas empresas e fora delas para que possam ser utilizados com segurança e contar com a velocidade adequada, sendo, assim, tratados em tempo real, sem perderem valor quando utilizados nos negócios. De acordo com Hieaux (2015), a Internet das Coisas aliada ao uso de Big Data dará base para uma economia de produtos e serviços personalizados, pois os consumidores terão o perfil mapeado e seus dados serão coletados e analisados, com isso, empresas ofertarão serviços cada vez mais customizados aos desejos e às necessidades de seus clientes. De acordo com dados da Internacional Data Corporation (2016), empresa que presta serviços de consultoria nas indústrias de TI, telecomunicações e mercados de consumo em massa de tecnologia em pesquisa comparativa feita na América Latina, a projeção de mercado da IoT em 2014 foi de USD 7 bilhões e, para 2020, a estimativa é de USD 15,6 bilhões.

A Radio Frequency Identification (RFID), ou na sua tradução direta "Identificação por Rádio Frequência", é um termo que descreve qualquer sistema de identificação no qual um dispositivo eletrônico que usa frequência de rádio para se comunicar é anexado a um item. Conforme apontam Glover e Bhatt (2007), a identificação por rádio frequência é uma tecnologia de comunicação que utiliza ondas de rádio, produzidas e enviadas por equipamentos eletrônicos para a identificação de produtos, documentos, objetos, animais e, até mesmo, seres humanos. Por intermédio dessa tecnologia, é possível o desenvolvimento de diversos sistemas de rastreamento e de monitoramento sem a necessidade de contato físico ou visual e, a partir de sua integração com a Internet das Coisas, novos serviços podem ser gerados para garantir maior segurança à população.

\subsection{A Segurança Pública no Brasil}

Da mesma forma que a instalação de câmeras de segurança em vias públicas tem se tornado uma prática corrente pelos órgãos públicos responsáveis pelo trânsito, novas ações de monitora- 
mento se encontram igualmente respaldadas pelo artigo 144 do texto constitucional, segundo o qual o Estado deve garantir segurança aos cidadãos por meio da execução de políticas públicas eficientes (BRASIL, 1988).

A criminalidade, principalmente a violenta e urbana, é um dos principais desafios mundiais e um dos grandes motivos de preocupação da sociedade brasileira. Tobar (2015) destaca que a própria mensuração da violência já se apresenta como tarefa árdua, seja por sua amplitude ou pela baixa disponibilidade e transparência de suas cifras.

Segundo Abramovay (2015), em 2014, não houve outro país do mundo, sem guerra declarada, em que mais seres humanos mataram outros seres humanos do que no Brasil. Conforme apresenta o Anuário Brasileiro de Segurança Pública (2015), apenas em 2014, no Brasil, foram ao menos 58.497 pessoas vítimas de mortes violentas. Registrou-se também, em 2014, o assassinato de 398 policiais, pelo menos um por dia. A cada 3 horas uma pessoa foi morta vítima de confrontos com a polícia naquele mesmo ano. Por outro lado, a população carcerária atingiu o patamar de 607.373 pessoas e o número de adolescentes em cumprimento de medida socioeducativas privativas de liberdade cresceu $443 \%$ nos últimos 17 anos. Os números apresentados denotam, por si só, a necessidade de um novo olhar sobre o atual modelo de Gestão da Segurança Pública brasileiro.

\subsection{Tecnologias Integradas à Segurança Pública}

Um interessante exemplo do uso da Internet das Coisas na Segurança Pública é trazido por Lemos et al. (2018), os autores apresentam a modernização progressiva dos mecanismos de segurança urbana em cidades brasileiras: Águas de São Pedro, no Estado de São Paulo, conta com um complexo de câmeras de alta resolução com tecnologia OCR (que conseguem reconhecer caracteres de placas de veículos) e tecnologia de reconhecimento facial, além de sensores dos mais variados tipos para auxiliar os agentes públicos na resolução de crimes e na manutenção da ordem.

Outro grande exemplo de uso da Internet das Coisas na Segurança Pública foi a aquisição de dispositivos de reconhecimento facial para testes pelo governo brasileiro em janeiro de 2019. Segundo a empresa de inteligência Alinger (2018), o grande benefício do uso de inteligências artificiais para segurança pública de forma preditiva é permitir que crimes sejam impedidos com as informações que os agentes públicos já terão em mãos. Tecnologias de reconhecimento facial são fundamentais para o acompanhamento de imagens em câmeras de alta resolução, fornecendo aos agentes as informações sobre suspeitos com comportamentos maliciosos, já que antes que eles entrem em ação são capturadas as imagens de momentos considerados suspeitos.

Lemos et al. (2018) também enfatizam que ao aplicar de forma concreta as noções de necessidade e de proporcionalidade, o Poder Público fica vedado a fazer uso dos dados coletados por equipamentos de segurança para as finalidades que sirvam de justificativa para sua coleta, o que não pode ser feita de forma arbitrária ou genérica. A regra geral da legislação brasileira diz que há a necessidade de obtenção do consentimento prévio e expresso dos indivíduos para o tratamento de seus dados pessoais. 


\section{Metodologia}

Este trabalho é constituído de uma pesquisa prospectiva e traz resultados direcionados ao uso de tecnologias no campo da Internet das Coisas que possam ser aplicadas à segurança pública no Brasil, com a prototipagem de sistemas envolvendo o reconhecimento facial, sensores de disparo e monitoramento de veículos que envolvem a tecnologia RFID. Foi realizada uma revisão de literatura, seguida de uma prospecção tecnológica dos três últimos anos, utilizando-se as bases patentárias Derwent Index Innovation, Questel-Orbit ${ }^{\circledR}$ e Lens, conforme apresentado no Quadro 1, que descreve as bases patentárias utilizadas na pesquisa:

Quadro 1 - Bases Patentárias utilizadas na busca

\begin{tabular}{|c|c|c|}
\hline IDENTIFICADOR & NomE & ENDEREÇO ELETRÔNICO \\
\hline 1 & Questel-Orbit $\mathbb{R}$ & https://www36.orbit.com/ \\
\hline 3 & Lens & https://www.lens.org/ \\
\hline 4 & Derwent & https://clarivate.com/products/derwent-world-patents-index/ \\
\hline
\end{tabular}

Fonte: Elaborado pelos autores deste artigo (2019)

De acordo com Kupfer e Tigre (2004), uma "prospecção tecnológica" pode ser definida como uma forma sistemática de mapear desenvolvimentos científicos e tecnológicos futuros capazes de influenciar de forma significativa uma indústria, a economia ou a sociedade. Diferentemente das atividades de previsão clássica, que se dedicam a antecipar um futuro suposto como único, os exercícios de prospecção são construídos a partir da premissa de que são vários os futuros possíveis. A prospecção tecnológica, utilizando informações oriundas dos documentos de patentes, tem se mostrado uma potente ferramenta e um instrumento bastante eficaz no apoio à tomada de decisão, tendo em vista o estado da arte disponível no seu conteúdo que permite identificar tecnologias relevantes, parceiros, concorrentes no mercado, rotas tecnológicas, inovações, investimentos, processos, produtos, PD\&I, fusões e aquisições, entre outras.

A pesquisa foi dividida em seis cruzamentos com temas-chave dentro da proposta tecnológica inicial nos dias 22/12/2018 e 09/01/2019. As pesquisas foram realizadas utilizando-se palauras de busca compostas de operadores boleanos contendo termos que correspondem às palavras-chave da pesquisa proposta.

\section{Análise e Discussão}

Por meio da metodologia descrita, foram analisados os documentos científicos (produção bibliométrica) e famílias de patentes (produção patentométrica), de maneira quantitativa, trazendo a apresentação de resultados da busca de documentos científicos e patentes, panorama da produção científica e tecnológica no mundo, principais instituições científicas e empresas depositantes, documentos por área partindo da proteção de dispositivos que operem dentro do conceito da Internet das Coisas aplicados à Segurança Pública e direcionando a pesquisa para três categorias de dispositivos. 


\subsection{Segurança Pública e Internet das Coisas}

Na primeira etapa da pesquisa foram relacionadas buscas por patentes de dispositivos e sistemas de monitoramento variados que envolvessem o uso da Internet das Coisas com aplicação dentro da segurança pública. O quadro de identificadores a seguir expõe as linhas criadas com o uso de operadores boleanos para busca de patentes dentro desse segmento da pesquisa:

Quadro 2 - A Internet das Coisas e a Segurança Pública

\begin{tabular}{|c|c|c|c|c|}
\hline LINHA & DESCRIÇÃO & LENS & QUESTEL-ORBIT & DERWENT \\
\hline 1 & $\begin{array}{c}\text { (PUBLIC SAFETY) OR ((PUBLIC } \\
\text { SECURITY) OR POLICE }\end{array}$ & 33039 & 22984 & 8626 \\
\hline 2 & $\begin{array}{c}\text { (SEGURANÇA PÚBLICA) OR (SEGURANÇA } \\
\text { PUBLICA) OR POLIÍCIA OR POLICIA }\end{array}$ & 1 & 231 & 0 \\
\hline 3 & $\begin{array}{l}\text { (IOT OR (INTERNET OF THINGS) } \\
\text { OR (INTERNET DAS COISAS)) AND } \\
\text { ((PUBLIC SAFETY) OR (PUBLIC } \\
\text { SECURITY) OR POLICE OR (SEGURANÇA } \\
\text { PÚBLICA) OR (SEGURANÇA PÚBLICA) } \\
\text { OR POLÍCIA OR POLIÍCIA) }\end{array}$ & 11070 & 526 & 3479 \\
\hline
\end{tabular}

Fonte: Elaborado pelos autores deste artigo (2019)

Para a palavra "public safety OR public security OR police", foram encontradas diversas aplicações de dispositivos e sistemas constituídos de patentes relativas a diversos sistemas de monitoramento não tripulados realizados por drones, fornecendo dados para a segurança local, sistemas de registro e mapeamento de crimes com localização GPS para registro geográfico dos tipos crimes ocorridos em local específico, sistemas de detecção facial e do movimento das mãos com câmeras sensíveis e algoritmos que reconhecem movimentos, botões de socorro automáticos por meio de localizador com sistema GPS com cadastro de usuário e acionamento imediato de polícia e bombeiros, sistemas de proteção a ataques on-line e demais dispositivos elencados a seguir:

a) Sistemas de Segurança D2D (Devide-To-Device) para acionamento automático de sistemas de segurança.

b) Sistemas de monitoramento integrados entre uma nuvem pública e um recurso local em uma rede privada, permitindo que uma conexão seja mais facilmente feita quando um aplicativo na nuvem pública acessa esse recurso local na rede privada criando um sistema de monitoramento compartilhado das ruas.

c) Serviços de controle interno de ambientes e gerenciamento integrado baseado em etiquetas RFID e Internet das Coisas.

d) Capacete inteligente utilizando a Internet das Coisas para envio de dados de trânsito e de monitoramento.

e) Sistemas de monitoramento de controle de tráfego.

Para a busca "(segurança pública) OR (seguranca publica) OR polícia OR policia", apenas foi encontrada uma patente na base Lens com conteúdo diverso do almejado nesta pesquisa. Na 
base Orbit, foram obtidos 231 resultados efetivos, mas, pela abrangência do tema, apenas duas tecnologias se destacam para a pesquisa, que foram: sistemas integrados de segurança pública e privada, em especial o desenvolvimento de um sistema de busca de pessoas e verificação da autenticidade de documentos de identificação por reconhecimento facial biométrico com validação pela leitura de dados do documento por proximidade, biometria digital e senha. $\mathrm{O}$ aplicativo pode ser projetado para encontrar pessoas desaparecidas, vítimas do crime e pessoas sendo procuradas pela polícia.

A busca "(iot OR (internet of things) OR (internet das coisas)) AND ((public safety) OR (public security) OR police OR (seguranca publica) OR (segurança pública) OR policia OR polícia)" é a linha mais complexa desta parte da pesquisa e, por esse motivo, muitas das 526 patentes encontradas já apontam dispositivos já citados, aos quais se acrescentou como resultados dentro do escopo da pesquisa:

a) Sistemas de identificação em prisões usando tecnologia etiquetas RFID.

b) Sistemas de monitoramento integrado para Smart Cities.

c) Sensores de movimento utilizando tecnologia da Internet das Coisas para envio de informações em tempo real para a polícia.

d) Sensores de disparo com alerta de evento crítico de arma de fogo.

e) Sistema de controle de acesso instalado na passagem da porta, contendo uma placa de identificação por radiofrequência (RFID) que está integrada na central de controle e unidade de controle de posicionamento.

f) Sistema de segurança predial baseado na Internet das Coisas, contendo câmara de monitoração de edifícios conectada com dispositivo de segurança e unidade de comunicação do sistema de controle de acesso;

Por ter maior quantidade de termos, a terceira linha foi utilizada para a apresentação gráfica de dados globais sobre a prospecção tecnológica realizada, relacionando a Internet das Coisas (IoT) e a Segurança Pública:

Figura 1 - Patentes por área tecnológica

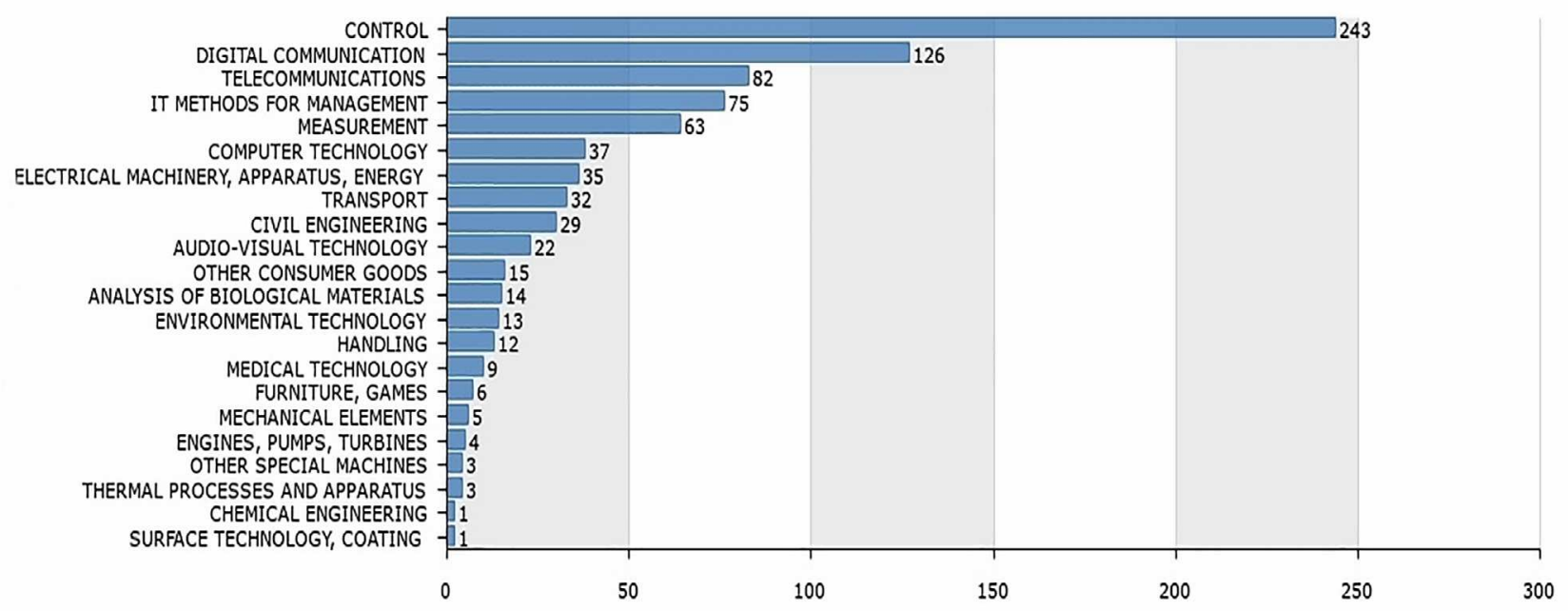

Fonte: Elaborada pelos autores deste artigo (2019) 
A Figura 1 apresenta os resultados do número de patentes por área tecnológica, apontando que as áreas de controle (243 patentes), comunicação digital (126 patentes) e telecomunicações (82 patentes) e mensuração (63 patentes) são as quatro áreas com maior quantidade de patentes desenvolvidas, apontando que a interação da Internet das Coisas é voltada para controle e comunicação automatizados.

Figura 2 - Empresas detentoras de famílias de patentes

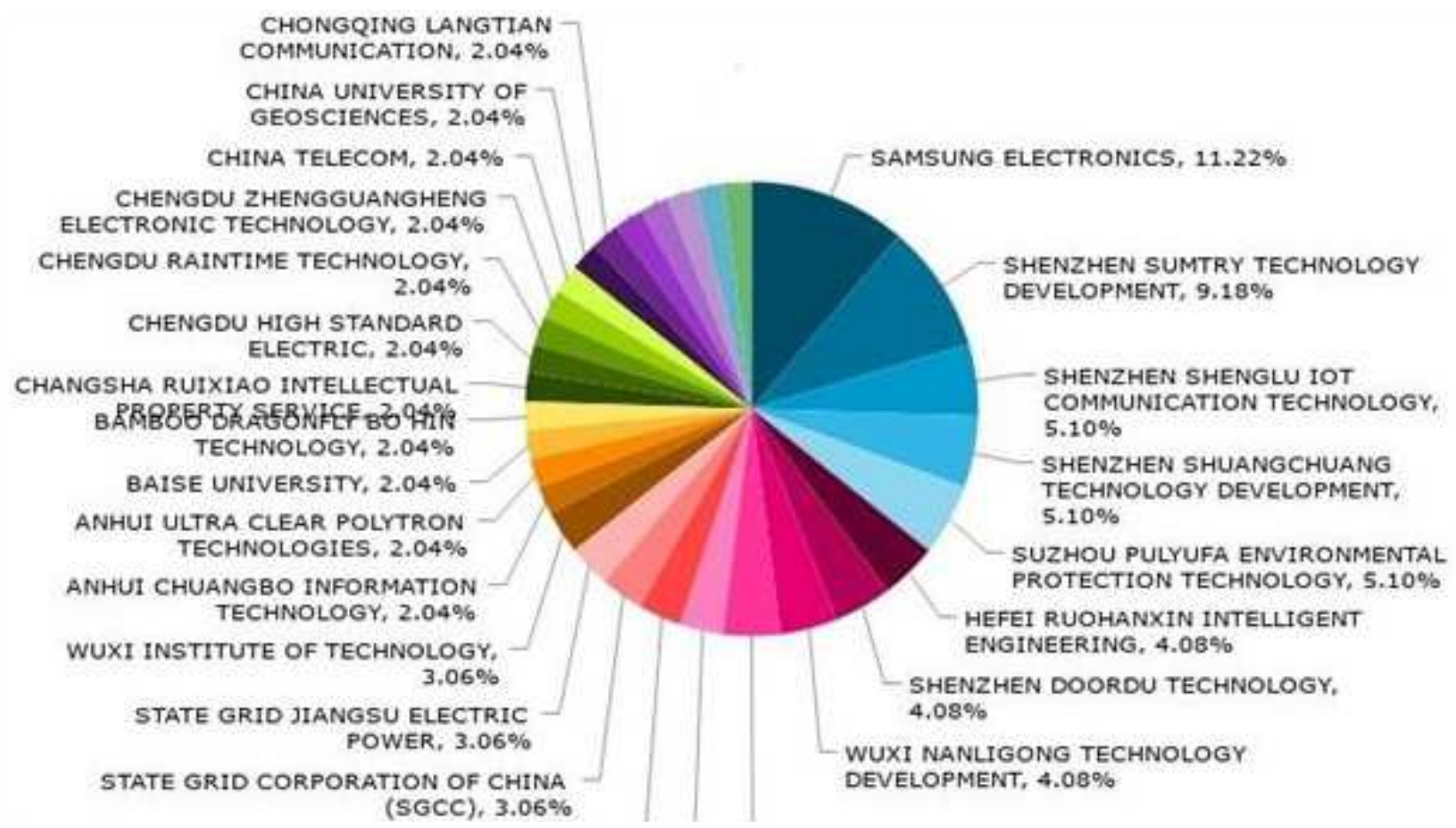

Fonte: Elaborada pelos autores deste artigo (2019)

A Figura 2 apresenta os resultados do número de patentes por meio das empresas que as desenvolveram. A Samsung Electronics detém 11,22\% das patentes registradas no mercado, sendo seguida pela Shenzhen Sumtry Technology Development com 9,18\% e sua correlata, a Shenzhen Shuangchuang Technology Development, se apresenta como detentora de 5,1\% das patentes registradas em bases mundiais.

Ao analisarmos as famílias de patentes por países de origem, é possível perceber o amplo destaque da China como detentora da maior quantidade de patentes registradas em bases mundiais, com 469 famílias de patentes, sendo seguida por países europeus, que, juntos, detêm apenas 35 famílias de patentes.

\subsection{Dispositivos de Reconhecimento Facial}

A segunda parte da pesquisa foi composta do cruzamento de dados sobre a Internet das Coisas (IoT) concatenada ao uso de dispositivos de reconhecimento facial para promoção da segurança pública. Vale salientar que, em 2019, o governo brasileiro adquiriu famílias de produtos chineses ligados a essa tecnologia, apontando que o reconhecimento facial é uma tecnologia prioritária nas novas diretrizes tecnológicas para a segurança pública nacional. Os termos utilizados foram montados de maneira a realizar uma pesquisa por famílias patentárias, relacionando os temas supracitados e os resultados obtidos na busca, que foram os seguintes: 
Quadro 3 - Internet das Coisas e Dispositivos de Reconhecimento Facial

\begin{tabular}{|c|c|c|c|c|}
\hline IDENTIFICADOR & Descrição & LENS & Questel-Orbit & Derwent \\
\hline 1 & $\begin{array}{l}\text { (internet of things) AND } \\
\text { (facial recognition) }\end{array}$ & 113 & 106 & 2 \\
\hline 2 & $\begin{array}{l}\text { ((internet of things) OR iot } \\
\text { OR (internet das coisas)) } \\
\text { AND ((facial recognition) OR } \\
\text { (reconhecimento facial)) }\end{array}$ & 1.526 & 209 & 2 \\
\hline 3 & $\begin{array}{l}\text { ((public safety) OR (public } \\
\text { security) OR police) AND ((facial } \\
\text { recognition) OR recognition) }\end{array}$ & 86.240 & 1.784 & 1.207 \\
\hline
\end{tabular}

Fonte: Elaborado pelos autores deste artigo (2019)

Com o uso da combinação "internet of things AND facial recognition" nas três bases patentárias pesquisadas, foram obtidos diversos modelos e diversas patentes de utilidade que apontam para novas tecnologias que integram os dois temas prioritários na busca, por exemplo, sistemas de destrancamento de portas, utilizando o reconhecimento facial e automaticamente realizando o envio dos dados para o proprietário com informações de localização e ativação de alarmes de segurança.

Foram encontrados diversos dispositivos de autenticação baseados no reconhecimento facial, utilizando método de controle inteligente da Internet das Coisas em que todos os usuários em uma área-alvo podem ser identificados de acordo com a prioridade do usuário. Também se verificou a existência de sistemas de monitoramento de segurança como um olho mágico eletrônico com tranca combinada com o reconhecimento facial de um usuário previamente cadastrado. A Figura 3 traz os resultados do cruzamento de dados envolvendo o reconhecimento facial e a Internet das Coisas de patentes da base Derwent Index Innovation:

Figura 3 - Famílias de patentes por área de conhecimento
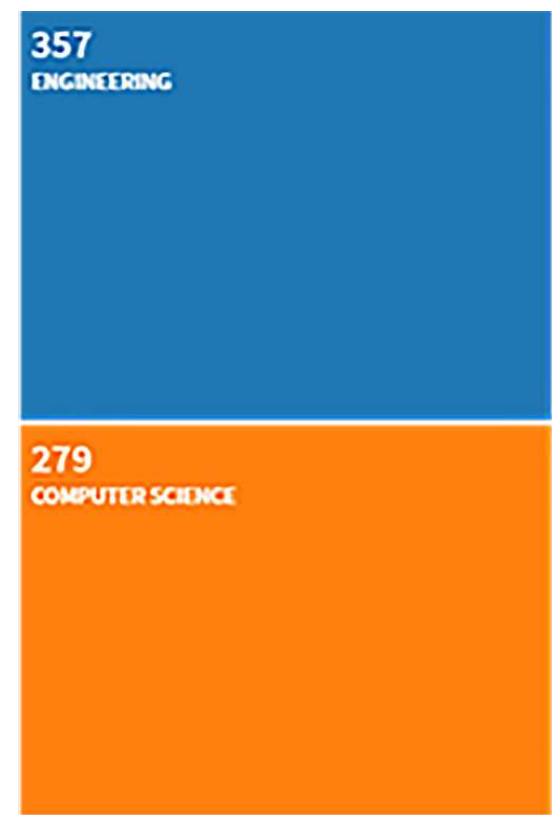
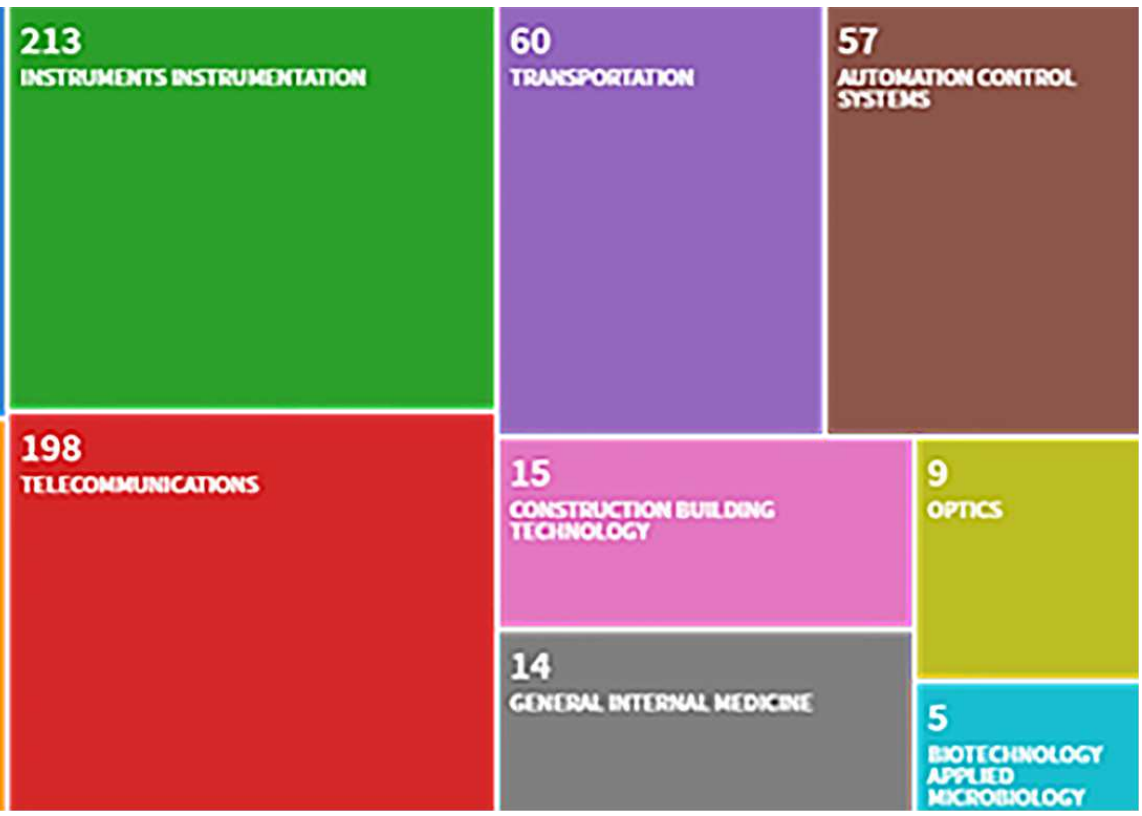

Fonte: Derwent Index Innovation (2018) 
Para a busca "((internet of things) OR iot OR (internet das coisas)) AND ((facial recognition) OR (reconhecimento facial))", foram encontrados alguns dispositivos que reúnem o reconhecimento digital e facial: uma das invenções encontradas divulga uma trava de combinação de impressão digital controlada por meio da Internet das Coisas. Outra tecnologia que se mostra como tendência dentro dessa linha de busca são os dispositivos de reconhecimento facial implantados em drones para segurança em eventos públicos. Por fim, essa parte da pesquisa também revelou que o mapeamento facial está sendo usado em larga escala para criação de sistemas operacionais de banco de dados policiais.

Na pesquisa "((public safety) OR (public security) OR police) AND ((facial recognition) OR recognition)", foram encontradas quase 90.000 patentes, que, depois de refinadas, extraiu-se os resultados já citados, a partir disso, foram encontrados os seguintes dispositivos dentro das aplicações propostas:

a) Sistema de busca de pessoas e verificação da autenticidade de documentos de identificação por reconhecimento biométrico facial com validação pela leitura de dados do documento por proximidade, biometria digital, senha ou outros meios.

b) Identificadores de emoções expressas facialmente e nos membros superiores por meio de algoritmo interpretando movimentos faciais e das mãos, criando um sistema que possa identificar tensões do usuário em uma situação de tensão ou depoimento.

c) Dispositivos para conferência de documentos pela face humana.

d) Sistema e método para análise de vídeo com base em campos de segurança pública. $\mathrm{O}$ método compreende as etapas de adquirir informação de imagem de face de uma informação de imagem de motorista e placa de um veículo quando um veículo é detectado em uma região predeterminada.

e) A invenção divulga um sistema de rastreamento de trajetória de veículo e reconhecimento de placa de veículo, compreendendo uma câmera em destaque disposta em cada ponto de monitoramento.

f) Óculos com sistema de reconhecimento facial com a integração de um algoritmo de modelagem de imagem 3D e um algoritmo de reconhecimento.

Por se tratar do cruzamento mais completo, a terceira linha de buscas gerou o quadro gráfico de dados sobre a concatenação da Internet das Coisas (IoT) com dispositivos de reconhecimento facial. Algoritmos com essa função estão sendo colocados em evidência dentro da segurança pública devido ao seu alto potencial para a solução de crimes com o uso de câmeras de segurança de alta resolução. 
Edilson Ponciano de Lima, João Paulo Lima Santos, Vinícius José Ferro Gomes, Erivaldo Oliveira de Matos, Francisco Medson Lima Maia, Luiz André da Silva Lima, José Maurício Alencar Filho, Wagner Gonçalves Oliveira e Silva, Gesyca Patrícia da Silva Santos

Figura 4 - Detentores de Famílias de Patentes

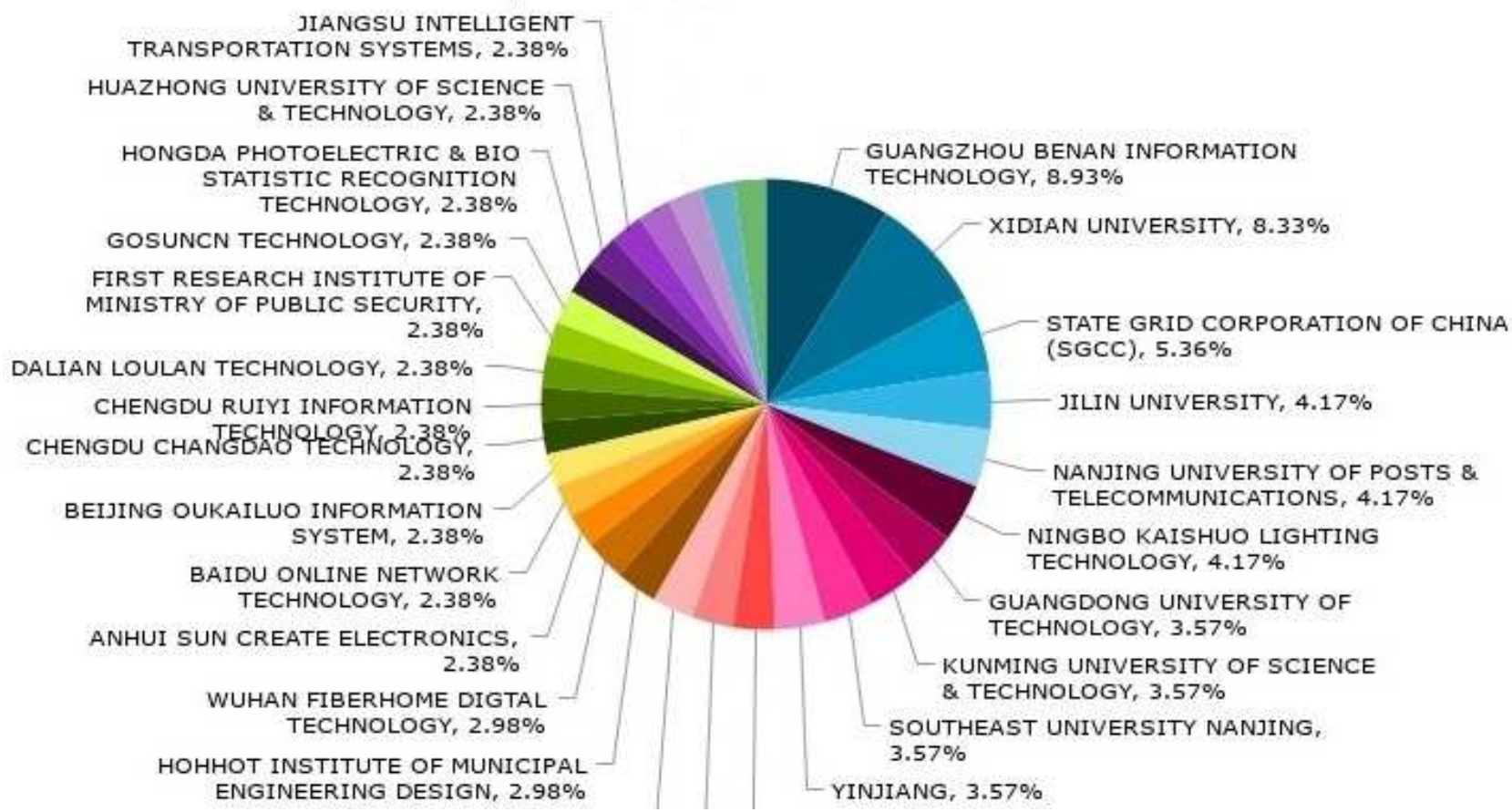

Fonte: Elaborada pelos autores deste artigo com base no Questel Orbit (2019)

A Figura 4 aponta que duas empresas e uma universidade da China são as maiores detentoras de patentes no mercado, a Guangzhou Benan Information detém quase $9 \%$ das patentes registradas, sendo seguida pela Xidian University, com 8,33\%, e pela State Grid Corporation of China com $5,36 \%$ das patentes registradas no mercado.

\subsection{Internet das Coisas e Sensores de Disparo}

O cruzamento de busca para dispositivos utilizando a Internet das Coisas e os sensores de disparo foi feito por meio dos seguintes termos:

Quadro 4 - Internet das Coisas e Sensores de Disparo

\begin{tabular}{|c|c|c|c|c|}
\hline LiNHA & DEscrição & LENS & Questel-Orbit & DERWENT \\
\hline 1 & $\begin{array}{l}\text { (iot OR (internet of things)) AND } \\
\text { ((trigger sensor) OR shot) }\end{array}$ & 948 & 231 & 32 \\
\hline 2 & (iot OR (internet das coisas)) AND (disparo OR tiro) & 4 & 1 & 0 \\
\hline 3 & $\begin{array}{l}\text { (public safety OR public security OR police) } \\
\text { AND (shot sensor OR shot OR sensor) }\end{array}$ & 174.522 & 7.216 & 954 \\
\hline 4 & $\begin{array}{c}\text { (seguranca publica) OR (segurança pública) } \\
\text { OR sensor AND (disparo OR tiro) }\end{array}$ & 34 & 23 & 1 \\
\hline 5 & $\begin{array}{l}\text { (iot OR (internet of things) OR (internet das } \\
\text { coisas)) AND ((public safety) OR (public security) } \\
\text { OR police OR (seguranca publica) OR (segurança } \\
\text { pública) OR policia OR polícia) AND (shot) }\end{array}$ & 472 & 18 & 1 \\
\hline
\end{tabular}

Fonte: Elaborado pelos autores deste artigo (2019) 
Apenas duas linhas de busca apresentaram resultados dentro do escopo da pesquisa: para a combinação "(iot OR (internet of things)) AND ((trigger sensor) OR shot)", a pesquisa traz patentes contendo uma série de dispositivos conectados à Internet das Coisas por comando de voz, como sensores de identificação de imagem e de som, apontando para um usuário cadastrado num banco de dados, bem como sistemas de segurança doméstica envolvendo monitoramento com vídeo, som e captura de imagens em tempo real.

Para a busca "(public safety OR public security OR police) AND (shot sensor OR shot OR sensor)", foi encontrado um único resultado que é de ideal interesse para a pesquisa, pois trata da criação de sistemas e de métodos para conectar câmeras privadas de vizinhança e realizar o monitoramento compartilhado de áreas públicas. Algumas das referências das patentes encontradas incluem o fornecimento de uma pluralidade de câmeras com cada um dos dispositivos ligados a um sistema de segurança respectivo, protegendo edifícios, detectando eventos de alarme em tempo real e garantindo o monitoramento em tempo real de eventos em toda a cidade. Para melhor incorporar os resultados desta etapa da pesquisa, os gráficos a seguir representam os dados encontrados em patentes que envolvem a integração entre sensores de disparo e a Internet das Coisas:

Figura 5 - Empresas detentoras de famílias de patentes



Fonte: Elaborada pelos autores deste artigo (2019)

A Figura 5 mostra que os cinco maiores detentores de patentes envolvendo sensores de disparos são corporações e universidades pertencentes à China.

Ao observamos a distribuição de patentes por áreas tecnológicas, percebe-se as áreas de controle, mensuração, transporte, maquinário elétrico e engenharia civil como as que detêm maior quantidade de dispositivos desenvolvidos. A Figura 6 apresenta os países detentores de famílias de patentes e aponta a China como a maior desenvolvedora de dispositivos envolvendo sensores de disparos: 
Figura 6 - Países detentores de famílias de patentes

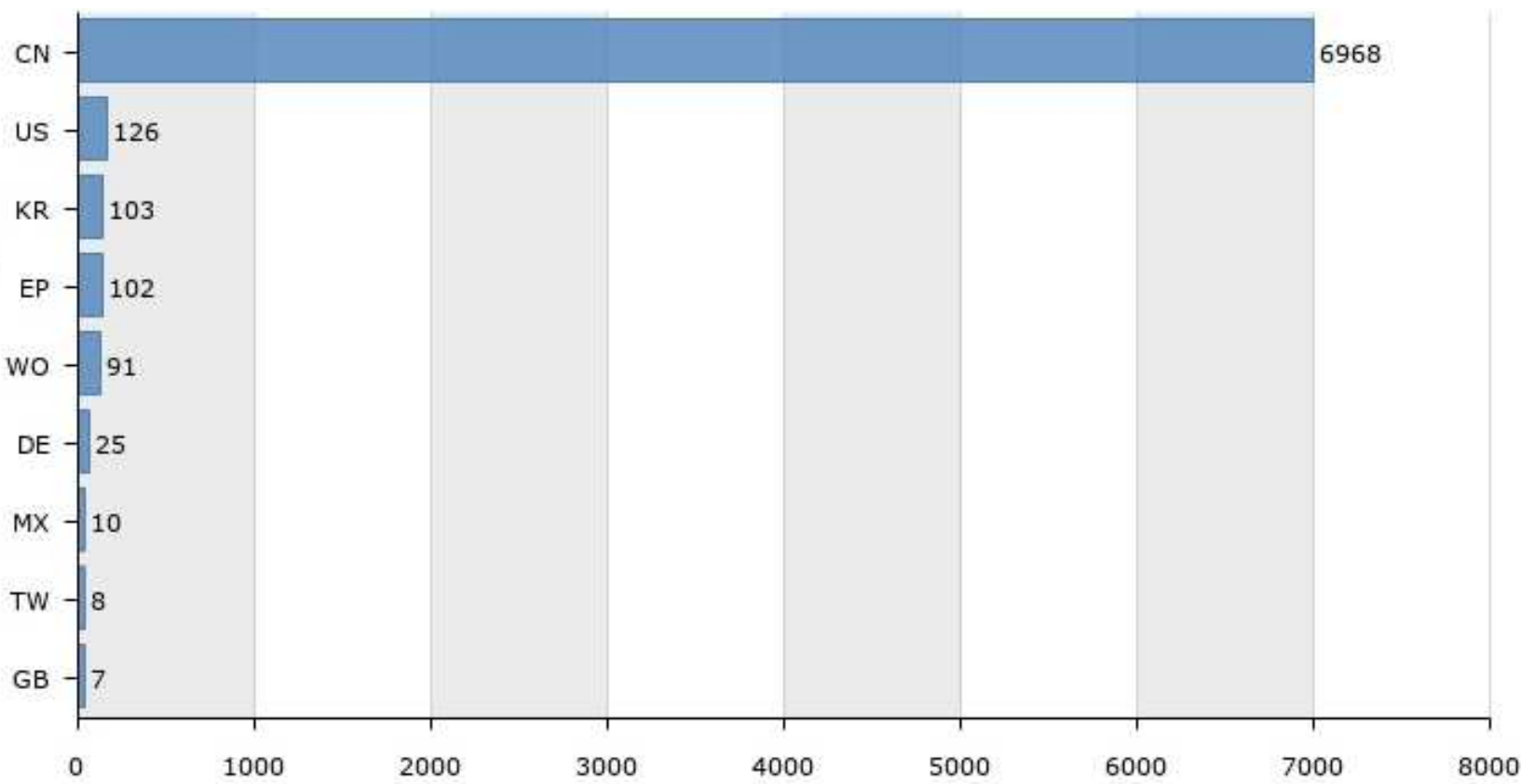

Fonte: Elaborada pelos autores deste artigo (2019)

Considera-se, em comparação com outros resultados obtidos na pesquisa, que há pouco aproveitamento de patentes neste segmento de atuação, pois sensores de disparos já possuem certo tempo de mercado e até já são usados com a finalidade proposta em algumas cidades. Outro dado importante é que a cidade do Rio de Janeiro já tentou implantar sistemas contendo sensores de disparo durante o ano de 2013, mas o projeto não foi bem-sucedido.

\subsection{Internet das Coisas e Radio-frequency Identification (RFID)}

Foram realizadas pesquisas relacionando a tecnologia RFID com a Internet das Coisas (IoT), segundo os seguintes termos e resultados:

Quadro 5 - Internet das Coisas e Radio-frequency Identification (RFID)

\begin{tabular}{|c|c|c|c|c|}
\hline LiNHA & Descrição & LENS & QUESTEL-ORBIT & DeRWENT \\
\hline 1 & $\begin{array}{c}\text { (iot OR (internet of things)) AND ((radio- } \\
\text { frequency identification) OR rfid) }\end{array}$ & 3.018 & 1.288 & 699 \\
\hline 2 & $\begin{array}{c}\text { ((radio-frequency identification) OR } \\
\text { rfid OR transponder) AND car }\end{array}$ & 36.979 & 1.306 & 903 \\
\hline 3 & $\begin{array}{c}\text { ((public safety) OR (public security)) } \\
\text { AND ((radio-frequency identification) } \\
\text { OR rfid OR transponder }\end{array}$ & 75.255 & 474 & 33 \\
\hline
\end{tabular}

Fonte: Elaborado pelos autores deste artigo (2019)

Para a linha "(iot OR internet of things) AND (radio-frequency identification OR rfid)", foram analisadas as patentes mais citadas e encontradas, dentro do escopo da pesquisa, diversas tecnologias envolvendo sistemas de rastreamento e de monitoramento de automóveis e de pessoas baseados na integração entre a Internet das Coisas (IoT) e a tecnologia RFID. Por exemplo, 
um sistema de gestão auxiliar de área turística que monitora todas as entradas e saídas de uma área turística empregando um método de informatização e de rastreio, contendo funções de cobrança automática, orientação turística e acomodação antecipada de aviso e melhorando o nível de gestão da área turística. As maiores detentoras de famílias de patentes contendo a tecnologia RFID concatenada a dispositivos de controle e de rastreamento que podem ser relacionados com a segurança pública são a Samsung Electronics Company Ltda., sendo seguida pela Semicondutor Energy Lab, Apple e IBM.

Para a combinação "((radio-frequency identification) OR rfid OR transponder) AND car", foram encontrados os melhores resultados dentro do escopo de patentes e modelos de utilidade relacionado à segurança pública, por isso, vale destacar as seguintes tecnologias:

a) Sistemas de monitoramento de bicicletas.

b) Dispositivos de controle de tráfego baseados em RFID.

c) Gerenciamento de sistemas de aluguel de carros.

d) Monitoramento de carros e conexão com mecânicos e oficinas.

e) Sistemas de alerta contra violação ou furto de bagagens.

f) Sistema de monitoramento em sinais de trânsito, possuindo módulos de identificação por radiofrequência (RFID) que estão localizados no próprio sinal de trânsito;

Para a linha "((public safety) OR (public security)) AND ((radio-frequency identification) OR rfid OR transponder", foram encontradas patentes e modelos de utilidade dentro do escopo da pesquisa com destaque para um método e sistema que supervisiona a sobrecarga em uma estrada com base em um "blockchain". O microprocessador recebe, então, o peso, a altura, a informação de dados de largura e o comprimento de um módulo de aquisição de dados. $\mathrm{O}$ sistema de gerenciamento de blockchain realiza a transmissão de dados com um sistema de gestão policial.

Figura 7 - Famílias de patentes por área de conhecimento

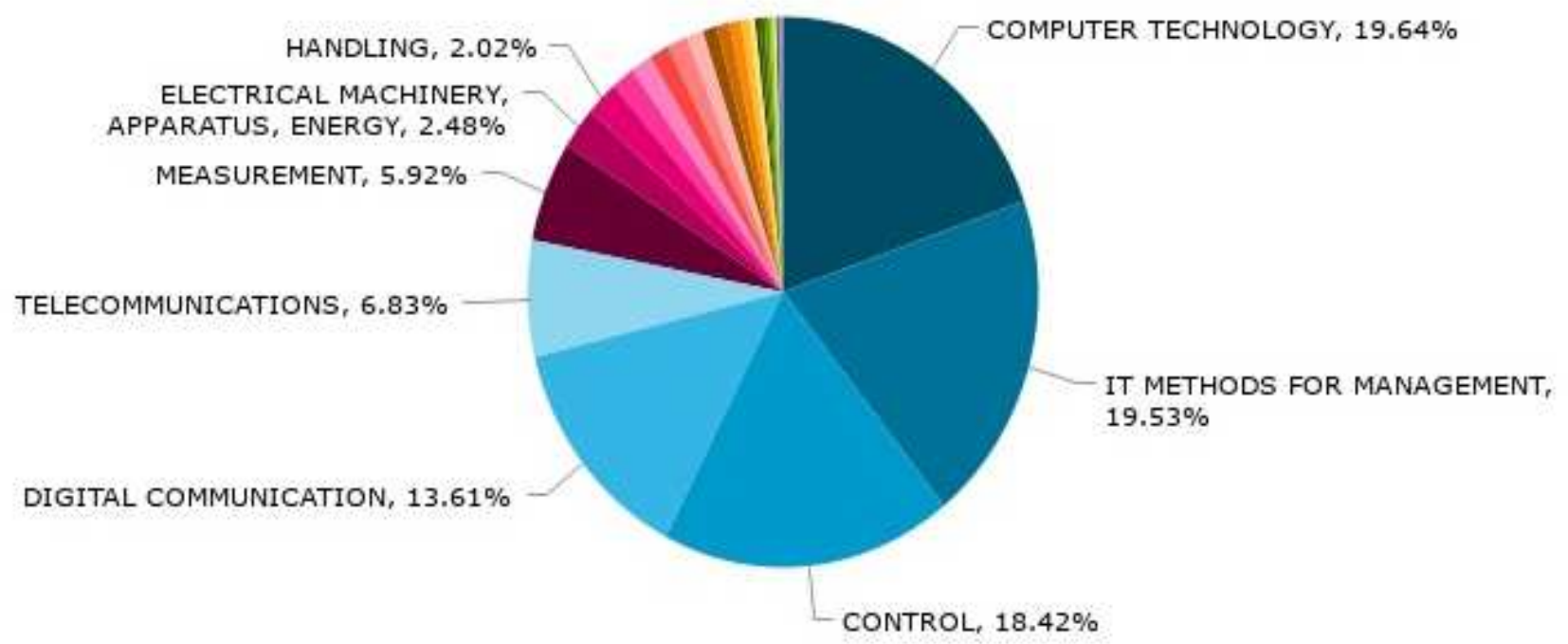

Fonte: Elaborada pelos autores deste artigo (2019) 
Por fim, percebe-se por meio da Figura 7 que as principais áreas de conhecimento contendo dispositivos patenteados envolvendo a tecnologia RFID estão ligadas às áreas de tecnologia da computação, de métodos de gerenciamento e de controle.

\section{Considerações Finais}

As inteligências artificiais podem se tornar fortes aliadas no uso da Internet das Coisas (IoT) e têm como base o intuito de facilitar e de agilizar a vida cotidiana do homem contemporâneo.

Com o aumento dos centros urbanos, os dados sobre a violência no cotidiano da sociedade se tornam cada vez mais preocupantes, e o uso de novas tecnologias para aprimorar os serviços da Segurança Pública torna-se essencial para a diminuição de situações conflituosas dentro das cidades. Os sistemas de monitoramento, de rastreamento, de socorro imediato e de identificação de indivíduos se mostram uma tendência dentro do mercado por meio do uso da Internet das Coisas (IoT).

Outrossim, sistemas de monitoramento e de registro de dados acabam por gerar situações que testam as fronteiras entre dois deveres constitucionais do Estado: o de garantir a segurança da população e o de assegurar seus direitos fundamentais, principalmente no que diz respeito ao direito à privacidade. Aquisições de tecnologia por parte do governo brasileiro vêm apontando que o reconhecimento facial é uma tecnologia prioritária nas novas diretrizes tecnológicas para a segurança pública nacional.

Pelos resultados obtidos na prospecção tecnológica, conclui-se que as inteligências artificiais, aliadas ao uso da Internet das Coisas (IoT), se tornam tendência dentro de um conjunto de dispositivos que poderão compor tecnologias ligadas à segurança pública e que diversos dispositivos, como sensores de disparo, tecnologia RFID e dispositivos de reconhecimento facial, podem ser combinados com a IoT, resultando em produtos e serviços capazes de solucionar crimes de forma preditiva, agilizar o atendimento em situações de urgência e aprimorar o gerenciamento do bem-estar social realizado pelos agentes de Segurança Pública.

\section{Referências}

ABRAMOVAY, Pedro. Um pacto para vencer nossa maior tragédia desde a escravidão. Anuário Brasileiro de Segurança Pública, Fórum Brasileiro de Segurança Pública, São Paulo, ano 9, p. 20-21, 2015. Disponível em: http://www.forumseguranca.org.br/produtos/anuario-brasileiro-deseguranca-publica/9o-anuario-brasileiro-de-seguranca-publica. Acesso em: 22 dez. 2019.

ALINGER. Colaboração tática: desafios de usar IoT e IA na segurança pública. 2018. Disponível em: https://www.aliger.com.br/blog/colaboracao-tatica-desafios-de-usar-iot-e-ia-na-segurancapublica/. Acesso em: 2 abr. 2019.

ANUÁRIO BRASILEIRO DE SEGURANÇA PÚBLICA. Fórum Brasileiro de Segurança Pública, São Paulo, ano 9, 2015. Disponível em: http://www.forumseguranca.org.br/produtos/anuario-brasileirode-seguranca-publica/9o-anuario-brasileiro-de-seguranca-publica. Acesso em: 22 jun. 2018.

BRASIL. Constituição da República Federativa do Brasil de 1988. Brasília, DF: Senado Federal, 1988. 
CHABERT, Jean Luc. A History of Algorithms: from the Pebble to the Microchip. Springer Verlag, 1999.

GLOVER, Bill; BHATT, Himanshu. Fundamentos de RFID. Rio de Janeiro: Alta Books, 2007.

HIEAUX, E. Big Data e Internet das coisas serão motores de uma nova economia. Junho de 2015. Disponível em: https://computerworld.com.br/2015/06/17/big-data-e-internet-das-coisas-seraomotores-de-uma-nova-economia/. Acesso em: 23 jun. 2018

INTERNATIONAL DATA CORPORATION. Previsão da IDC para o mercado de TIC no Brasil em 2016 aponta crescimento de 2,6\%. 2016. Disponível em: http://br.idclatin.com/releases/news. aspx?id=1970. Acesso em: 26 mar. 2019.

KUPFER, D.; TIGRE, P. B. Modelo SENAI de prospecção: documento metodológico. Capítulo 2: prospecção tecnológica. In: ORGANIZACION INTERNACIONAL DEL TRABAJO CINTERFOR. Papeles de La Oficina Técnica. Montevideo: OIT/CINTERFOR, 2004. n. 14.

LEMOS, Ronaldo et al. Internet das Coisas e Segurança Pública. 2018. Disponível em: https:// www.jota.info/opiniao-e-analise/artigos/internet-das-coisas-e-seguranca-publica-17042018. Acesso em: 24 maio 2018.

OLIVEIRA, André Henrique et al. Aplicações de Automação em IoT - Internet of Things. Revista Científica da FAEX, [S.l.], 2016.

STROTHER, Neil. Internet of Things. Navigant Research. 2016. Disponível em: https://www. navigantresearch.com/tag/internet-of-things. Acesso em: 22 maio 2018.

TAURION, Cezar. Big Data. São Paulo: Editora Brasport, 2013.

TOBAR, Felipe Salazar. Tendências criminais sul-americanas em perspectiva comparada. Revista Brasileira de Segurança Pública, São Paulo, 17. ed., Fórum Brasileiro de Segurança Pública, v. 9, n. 2, p. 88-109, ago.-set. 2015. Disponível em: http://www.forumseguranca.org.br/produtos/revistabrasileira-de-seguranca-publica/17a-edicao. Acesso em: 24 maio 2018.

VELASCO, Rodrigo Guerrero. An Antidote to Murder: city leaders across the Americas are exploiting science to reduce homicide. Outubro de 2015. Disponível em: http://salud.univalle.edu.co/ comunicandosalud/wp-content/uploads/2015/09/An-antidote-to-murder-Guerrero-Velasco-2015-1. pdf. Acesso em: 30 jun. 2018.

VERMA, N. et al. National Informatics Centre of India: E-government Tool Kit for Developing Countries. New Delhi: Unesco, Asia Pacific Regional Bureau for Communication and Information: National Informatics Centre, Dept. of Information Technology, Govt. of India, 2005. Disponível em: http://unesdoc.unesco.org/images/0013/00. Acesso em: 24 maio 2018.

\section{Sobre os Autores}

\section{Edilson Ponciano de Lima}

E-mail: edilsonponciano@yahoo.com

Mestre em Propriedade Intelectual e Transferência de Tecnologia para a Inovação.

Endereço profissional: Rua Carlos Povina Cavalcante, n. 17, ap. 511, Jatiúca, Maceió, AL. CEP: 57036-580. 
Edilson Ponciano de Lima, João Paulo Lima Santos, Vinícius José Ferro Gomes, Erivaldo Oliveira de Matos, Francisco Medson Lima Maia, Luiz André da Silva Lima, José Maurício Alencar Filho, Wagner Gonçalves Oliveira e Silva, Gesyca Patrícia da Silva Santos

\section{João Paulo Lima Santos}

E-mail: jpls@lccv.ufal.br

Doutor em Engenharia Civil.

Endereço profissional: Campus A. C. Simões, Av. Lourival Melo Mota, s/n, Tabuleiro do Martins, Maceió, AL. CEP: 57072-970.

\section{Vinícius José Ferro Gomes}

E-mail: viniciusferro_8@hotmail.com

Mestre em Propriedade Intelectual e Transferência de Tecnologia para a Inovação.

Endereço profissional: Centro Universitário Mario Pontes Jucá, UMJ, Av. Pres. Roosevelt, n. 1.200, Barro Duro, Maceió, AL. CEP: 57040-600.

\section{Erivaldo Oliveira de Matos}

E-mail: cont.matos@gmail.com Mestre em Propriedade Intelectual e Transferência de Tecnologia para a Inovação.

Endereço profissional: Av. Pres. Getúlio Vargas, n. 444. Sala 9, Serraria, Maceió, AL. CEP: 57046-140.

\section{Francisco Medson Lima Maia}

E-mail: medson.maia@fabricadebot.com

Especialista em Gerenciamento de Projetos.

Endereço profissional: Trav. Elias Ramos de Araújo, n. 30A, Quadra A, Bairro de Cruz das Almas, Maceió, AL. CEP: $57025-000$.

\section{Luíz André da Silva Lima}

E-mail: luiz.andre@iqb.ufal.br

Mestre em Propriedade Intelectual e Transferência de Tecnologia para a Inovação

Endereço profissional: Rua Pedro Américo, n. 18, Poço, Maceió, AL. CEP: 57025-890.

\section{José Maurício Alencar Filho}

E-mail: mauricioalencarf@gmail.com

Graduação em Engenharia Mecatrônica.

Endereço profissional: Rua José Pimentel leite passos, n. 70, Trapiche da Barra, Maceió, AL. CEP: 57010-740.

\section{Wagner Gonçalves e Silva}

E-mail:wagner.wgos@gmail.com

Graduação em Engenharia Mecatrônica.

Endereço profissional: Rua Desembargador Tenório, n. 225, Apto 202, Farol, Maceió, AL. CEP: 57050-050.

\section{Gesyca Patrícia da Silva Santos}

E-mail: gesycasantos13@gmail.com

Bacharela em Geografia.

Endereço profissional: Rua Alzira de Almeida Silva, s/n, Jacarecica, ap. 304, BL 15, Maceió, AL. CEP: 57038-635. 